\title{
Agentes ligantes e seus efeitos em concretos refratários alumina-magnésia
}

\section{(Binders and their effects on alumina-magnesia refratory castables)}

\author{
M.A.L. Braulio ${ }^{I}$ D. H. Milanez ${ }^{I}$, E.Y.Sako ${ }^{I}$,L.R.M. Bittencourt ${ }^{2}$, V.C.Pandolfelli ${ }^{I}$ \\ ${ }^{I}$ Grupo de Engenharia de Microestrutura de Materiais - GEMM, Departamento de Engenharia de Materiais, \\ Universidade Federal de S. Carlos, Rod. Washington Luiz, km 235, C.P. 676, S. Carlos, SP, 13565-905 \\ ${ }^{2}$ Magnesita S. A., Centro de Pesquisas e Desenvolvimento, Praça Louis Ensch 240, Contagem, MG \\ mariana_alb@yahoo.com.br,vicpando@power.ufscar.br
}

\begin{abstract}
Resumo
Concretos refratários alumina-magnésia são comumente ligados por cimentos de aluminato de cálcio. Estes materiais apresentam, em temperaturas elevadas, uma reação de caráter expansivo decorrente da espinelização in-situ $\left(\mathrm{MgAl}_{2} \mathrm{O}_{4}\right)$. Quando ligados por cimentos, reações adicionais (formação de $\mathrm{CA}_{2}$ e $\mathrm{CA}_{6}$ ) contribuem para a expansão. Uma alternativa para controlar a estabilidade volumétrica é a substituição do cimento tipicamente utilizado ( 30\%-p CaO) por outro de menor teor de cálcio ( 20\%-p CaO) ou por alumina hidratável. Tais substituições podem afetar as propriedades dos concretos, como a resistência mecânica a frio, a hidratação da magnésia e as propriedades durante e após a sinterização. Deste modo, o objetivo deste trabalho é avaliar o impacto destes ligantes no processamento de concretos alumina-magnésia. Sendo assim, pretende-se utilizar a fonte de ligante como uma ferramenta para a obtenção de concretos refratários com expansão engenheirada, visando-se aumentar a flexibilidade na seleção de materiais aplicados em panelas de siderurgia.
\end{abstract}

Palavras-chave: cimento de aluminato de cálcio, alumina hidratável, concretos alumina-magnésia.

Abstract

The usual binder of alumina-magnesia castables is the calcium aluminate cement. Due to in-situ spinel $\left(\mathrm{MgAl}_{2} \mathrm{O}_{4}\right)$ formation at high temperatures, these materials present an expansive behavior. When bonded with cement, further reactions $\left(\mathrm{CA}_{2}\right.$ and $C \mathrm{~A}_{6}$ formation) also contribute to the overall expansion. Changing the most common cement used $(\sim 30 \mathrm{wt} . \% \mathrm{CaO})$ for other containing less calcia $(\sim 20 \mathrm{wt} . \% \mathrm{CaO})$ or for hydratable alumina are suitable alternatives for controlling the castables' volumetric stability. Nevertheless, the binder replacement may affect castables properties, such as cold mechanical strength, MgO hydration degree and properties during and after sintering. Therefore, the objective of the present paper is to analyze the effects of these binders on the aluminamagnesia castables processing. As a result, the binder systems can be used as a tool for designing the alumina-magnesia castables expansion, increasing the flexibility on the selection of steel ladles linings.

Keywords: calcium aluminate cement, hydratable alumina, alumina-magnesia castables.

\section{INTRODUÇÃO}

Agentes ligantes de pega hidráulica são amplamente empregados nos concretos refratários. Entre eles, destacamse os cimentos de aluminato de cálcio e as aluminas hidratáveis. Dois aspectos relevantes nestes materiais são: as reações de hidratação que se desenvolvem durante a cura do material e as reações envolvidas durante sua decomposição até as temperaturas de aplicação. Os cimentos de aluminato de cálcio (CAC) estão disponíveis em composições distintas, sendo os mais comuns aqueles contendo 70 ou 80\%-p de alumina (30 ou 20\%-p de cálcia). As principais fases mineralógicas anidras presentes no cimento são o $\mathrm{CA}, \mathrm{CA}_{2}$ e $\mathrm{C}_{12} \mathrm{~A}_{7}$ (onde $\mathrm{C}=\mathrm{CaO}$ e $\mathrm{A}=\mathrm{Al}_{2} \mathrm{O}_{3}$ ), sendo responsáveis pela pega hidráulica observada nos CACs [1]. A quantidade de cada uma dessas fases varia entre os cimentos comerciais existentes. Diversas fases hidratadas são formadas pela reação da água com estes aluminatos de cálcio. Os hidratos predominantes são: $\mathrm{CAH}_{10}(\mathrm{CaO}$.
$\left.\mathrm{Al}_{2} \mathrm{O}_{3} \cdot 10 \mathrm{H}_{2} \mathrm{O}\right), \mathrm{C}_{2} \mathrm{AH}_{8}\left(2 \mathrm{CaO} \cdot \mathrm{Al}_{2} \mathrm{O}_{3} \cdot 8 \mathrm{H}_{2} \mathrm{O}\right), \mathrm{C}_{3} \mathrm{AH}_{6}(3 \mathrm{CaO}$. $\left.\mathrm{Al}_{2} \mathrm{O}_{3} \cdot 6 \mathrm{H}_{2} \mathrm{O}\right)$ e $\mathrm{AH}_{3}\left(\mathrm{Al}_{2} \mathrm{O}_{3} \cdot 3 \mathrm{H}_{2} \mathrm{O}\right)$ [1]. O tipo e a quantidade de hidratos formados dependem das fases mineralógicas do CAC, da razão cimento/água e da temperatura da reação. A temperatura de cura é o parâmetro que mais influencia no tipo de hidrato formado. Temperaturas superiores a $40{ }^{\circ} \mathrm{C}$ resultam em $\mathrm{C}_{3} \mathrm{AH}_{6}$ e o $\mathrm{AH}_{3}$, que são as fases hidratadas do cimento de maior estabilidade nesta temperatura, resultando em um desenvolvimento mais acelerado de resistência mecânica [2].

A secagem de concretos contendo cimento deve ser realizada de forma cautelosa, iniciando-se com um patamar a $110{ }^{\circ} \mathrm{C}$ para a remoção da água livre do concreto e para a completa conversão das fases metaestáveis do cimento [3]. Com o aumento da temperatura, ocorre a decomposição das fases hidratadas do cimento, até o reaparecimento de aluminatos de cálcio em temperaturas próximas a $600{ }^{\circ} \mathrm{C}$. Elevando-se ainda mais a temperatura, voltam a serem formadas as fases $\mathrm{CA}$ e $\mathrm{CA}_{2}$. Em temperaturas de 1400- 
$1500{ }^{\circ} \mathrm{C}$, o $\mathrm{CA}_{2}$ presente reage com a alumina do concreto, gerando a fase $\mathrm{CA}_{6}$, de elevada refratariedade. Devido a estas transformações, concretos contendo CAC apresentam excelente resistência mecânica após a secagem a $110{ }^{\circ} \mathrm{C}$, seguida por uma queda de resistência em temperaturas intermediárias [4].

Em concretos contendo microssílica o uso de cimento causa efeitos colaterais, devido à formação de fases de baixa refratariedade no sistema $\mathrm{CaO}-\mathrm{Al}_{2} \mathrm{O}_{3}-\mathrm{SiO}_{2}$, como a guelenita e a anortita [5]. Com um teor de $\mathrm{CaO}$ inferior a $0,1 \%$-p, ligantes compostos por aluminas hidratáveis possibilitam ao concreto a melhoria da resistência à corrosão e das propriedades a quente, levando a um desempenho superior em aplicações onde a presença de $\mathrm{CaO}$ não é tolerada [6].

Os produtos comerciais de aluminas hidratáveis baseiam-se na capacidade de re-hidratação da aluminarho ( $\rho)$, uma fase metaestável de pega hidráulica na temperatura ambiente. Esta alumina de transição reage com a água resultando na formação de baierita $\left(\mathrm{Al}_{2} \mathrm{O}_{3} \cdot 3 \mathrm{H}_{2} \mathrm{O}\right)$ e boemita gel $\left(\mathrm{Al}_{2} \mathrm{O}_{3} \cdot 1-2 \mathrm{H}_{2} \mathrm{O}\right)$ [7], sendo que a formação destes géis após a secagem é responsável pela resistência mecânica a verde neste sistema. Esta reação de hidratação é bastante sensível à temperatura de cura do concreto, sendo pouco intensa em baixas temperaturas $\left(<5^{\circ} \mathrm{C}\right)$, mas ativada em temperaturas mais elevadas $\left(>30^{\circ} \mathrm{C}\right)$ [7]. A presença de compostos específicos pode influenciar a reação de hidratação da alumina-rho. Em concretos contendo magnésia, este óxido reage com a alumina resultando na formação de hidrotalcita [8], acelerando a cura do concreto e alterando propriedades como a resistência mecânica a verde. Uma vantagem das aluminas hidratáveis é que estas não necessitam de um ambiente úmido durante a cura para desenvolver sua resistência mecânica. No entanto, estes ligantes requisitam maiores tempos de mistura e ocasionam uma baixa permeabilidade do concreto, sendo sensíveis a etapas subseqüentes do processamento, tal como a secagem. Devido a estes aspectos, a secagem destes materiais deve ser controlada para se evitar riscos de explosão durante o aquecimento inicial $\left(100-300{ }^{\circ} \mathrm{C}\right)$ [9]. Outra característica importante relacionada às aluminas hidratáveis é o seu comportamento mecânico em temperaturas intermediárias. Estudos recentes apontam para um decréscimo de resistência mecânica em temperaturas próximas a $1000{ }^{\circ} \mathrm{C}$ [10], o que pode ocasionar, durante o aquecimento, a geração de trincas associadas às tensões térmicas geradas. Esta queda de resistência mecânica possivelmente está associada à desidratação da baierita e da boemita $\left(200-550^{\circ} \mathrm{C}\right)$ e também as subseqüentes transformações de fases meta-estáveis da alumina $\left(600-1100{ }^{\circ} \mathrm{C}\right)$. Em temperaturas elevadas, os concretos alumina-magnésia apresentam o desenvolvimento de uma fase expansiva, o espinélio formado in-situ $\left(\mathrm{MgAl}_{2} \mathrm{O}_{4}\right)[11,12]$. Adicionalmente, a presença de cimento de aluminato de cálcio resulta no desenvolvimento de hexaluminato de cálcio $\left(\mathrm{CA}_{6}\right)$, reação de caráter expansivo que também contribui para a variação dimensional deste tipo de concreto [11].

Deste modo, o objetivo deste trabalho é avaliar o efeito de ligantes distintos (dois cimentos de purezas distintas, contendo 70 e $80 \%$-p de alumina, e uma alumina hidratável) no processamento de concretos alumina-magnésia, visandose a obtenção de condições alternativas para o controle da expansão deste material.

\section{MATERIAIS E MÉTODOS}

Três ligantes distintos foram selecionados e adicionados aos concretos em um teor de 6\%-p: os cimentos de aluminato de cálcio Secar 71, contendo 30\%-p de $\mathrm{CaO}$ (30C), e Secar Plenium, contendo 20\%-p de $\mathrm{CaO}$ (20C) (Kerneos, França) e uma alumina hidratável, o AlphaBond300 (Almatis, Alemanha), isento de $\mathrm{CaO}(0 \mathrm{C})$. Concretos vibrados alumina-magnésia tiveram sua distribuição de partículas projetadas por meio do modelo de empacotamento de Alfred e $\mathrm{q}=0,26$. A matriz de tais materiais (matérias-primas finas) foi composta por 6\%-p de sínter de magnésia (95\%p $\mathrm{MgO}, \mathrm{CaO} / \mathrm{SiO}_{2}=0,37$, Magnesita S. A., Brasil), 1\%-p de microssílica (971 U, Elkem, Noruega), 7\%-p de alumina reativa (CL370, Almatis, EUA) e $15 \%$-p de alumina tabular fina ( $d \leq 200 \mu \mathrm{m}$, Almatis, Alemanha). Para complementar as composições, aluminas tabulares grosseiras foram utilizadas como agregados ( $\mathrm{d} \leq 6 \mathrm{~mm}$, Almatis, Alemanha). O teor de água mínimo obtido para garantir uma moldagem adequada sob vibração foi de 3,9\%-p para a composição $30 \mathrm{C}, 4,8 \%$-p para a composição 20C e 5,3\%-p para a composição 0C.

Corpos de prova foram preparados em moldes cilíndricos (40 mm de diâmetro e de altura) e avaliados após a cura em $50{ }^{\circ} \mathrm{C}$, em ambiente saturado (no caso do cimento de aluminato de cálcio) e em ambiente insaturado (para a alumina hidratável), por intervalos distintos de tempo (1, 3, 5 e 7 dias). A resistência mecânica também foi avaliada após a secagem $\left(1\right.$ dia de cura a $50{ }^{\circ} \mathrm{C}$ e um dia de secagem a $\left.110^{\circ} \mathrm{C}\right)$ e em temperaturas intermediárias $(350,600,800$ e $\left.1000{ }^{\circ} \mathrm{C}\right)$. O ensaio utilizado foi de compressão diametral, realizado em uma máquina de teste universal (MTS Systems Corp., modelo 810, EUA). Foram rompidas cinco amostras por composição, a uma taxa de aplicação de carga constante de $42 \mathrm{~N} / \mathrm{s}$. A tensão de ruptura $\left(\sigma_{\mathrm{R}}-\mathrm{Pa}\right)$ é calculada conforme a equação:

$$
\sigma_{\mathrm{R}}=2 \cdot\left(\frac{P_{\text {máx }}}{\pi \cdot L \cdot D}\right)
$$

onde $\mathrm{P}_{\text {máx }}$ é a força de ruptura (N), Lé a altura e $\mathrm{D}$ o diâmetro do corpo de prova $(\mathrm{mm})$.

Paralelamente, a análise termogravimétrica foi realizada após 1 dia de cura dos concretos a $50{ }^{\circ} \mathrm{C}$, visando-se analisar a existência ou não de picos associados à decomposição de brucita. Estes ensaios de secagem foram efetuados a uma taxa de $10{ }^{\circ} \mathrm{C} / \mathrm{min}$ até $800{ }^{\circ} \mathrm{C}$. Foram coletados dados de massa e temperatura da amostra, registrados por computador em intervalos de $5 \mathrm{~s}$. A perda de massa durante a secagem foi avaliada por meio do parâmetro $\mathrm{W}$ e sua derivada $(\mathrm{dW} / \mathrm{dt})$, que indica a taxa de secagem, conforme as equações: 


$$
\begin{aligned}
& \mathrm{W}(\%)=100 \cdot\left(\frac{\mathrm{M}_{0}-\mathrm{M}}{\mathrm{M}_{0}-\mathrm{M}_{\mathrm{f}}}\right) \\
& \frac{\mathrm{dW}}{\mathrm{dt}}(\% / \mathrm{min})=\frac{\Delta \mathrm{W}}{\Delta \mathrm{t}}
\end{aligned}
$$

onde $\mathrm{W}$ é a perda de água que ocorre durante o aquecimento em relação à massa final do concreto seco $\left(\mathrm{M}_{\mathrm{f}}\right)$; $\mathrm{M}$ é a massa instantânea medida no tempo $\mathrm{t}_{\mathrm{i}}$ e $\mathrm{M}_{\mathrm{o}}$ a massa inicial.

Posteriormente, corpos de prova na forma de barras (25 $\mathrm{mm}$ x $25 \mathrm{~mm}$ x $150 \mathrm{~mm}$ ) foram preparados e caracterizados mecanicamente por meio do ensaio de flexão 3 pontos (MTS 810 , EUA), após a sinterização (taxa de aquecimento de 1 ${ }^{\circ} \mathrm{C} /$ min e patamar de $5 \mathrm{~h}$ a 1150,1300 e $1500{ }^{\circ} \mathrm{C}$.

$\mathrm{O}$ acompanhamento da espinelização in-situ e da formação de $\mathrm{CA}_{6}$ (para concretos contendo CAC) foi realizado pela análise da variação linear observada no ensaio de sinterabilidade assistida, por meio do equipamento Netzsch RUL 421E. Foram preparadas amostras cilíndricas $(\mathrm{h}=\mathrm{d}=50 \mathrm{~mm})$ com um furo central de $12,4 \mathrm{~mm}$. O ensaio foi efetuado a uma taxa de $3{ }^{\circ} \mathrm{C} / \mathrm{min}$ até a temperatura de $1500{ }^{\circ} \mathrm{C}$, sob carga compressiva de $0,02 \mathrm{MPa}$, sendo que as amostras permaneceram nesta temperatura por $5 \mathrm{~h}$. Os testes foram realizados em amostras verdes (pré-calcinadas a $600{ }^{\circ} \mathrm{C}$ por $5 \mathrm{~h}$ ), visando-se o estudo da formação de fases e sinterização do material. De modo complementar, a taxa de expansão foi obtida, pela derivada das curvas de expansão, indicando a velocidade de desenvolvimento das reações e a capacidade do concreto acomodar tais reações expansivas.

\section{RESULTADOS E DISCUSSÃO}

A variação do tipo de ligante afetou o comportamento de resistência mecânica durante a cura dos concretos alumina-magnésia. Como indica a Fig. 1, o concreto ligado por alumina hidratável (0C) apresentou, até o quinto dia, resultados superiores quando comparados ao concreto contendo CAC com maior teor de $\mathrm{CaO}$ (30C). Este aspecto pode ser associado com a reação entre a alumina e a magnésia, resultando na formação de hidrotalcita [8]. No entanto, tal reação também é expansiva e não foi suficiente para controlar a hidratação do $\mathrm{MgO}$ e, do quinto para o sétimo dia de cura, observou-se uma queda sutil de resistência mecânica, indicando a necessidade de cuidados nas condições de processamento de concretos ligados por aluminas hidratáveis. Quanto ao concreto contendo CAC com menor teor de $\mathrm{CaO}$ (20C), a resistência mecânica durante a cura foi sensivelmente inferior, uma vez que a quantidade de cálcio está diretamente associada a formação de aluminatos hidratados. Adicionalmente, seu teor de água (4,8\%-p) foi muito superior ao do concreto C30 (3,9\%-p), o que certamente resulta na geração de uma maior quantidade de poros neste material.

Com relação à resistência à hidratação da magnésia, a

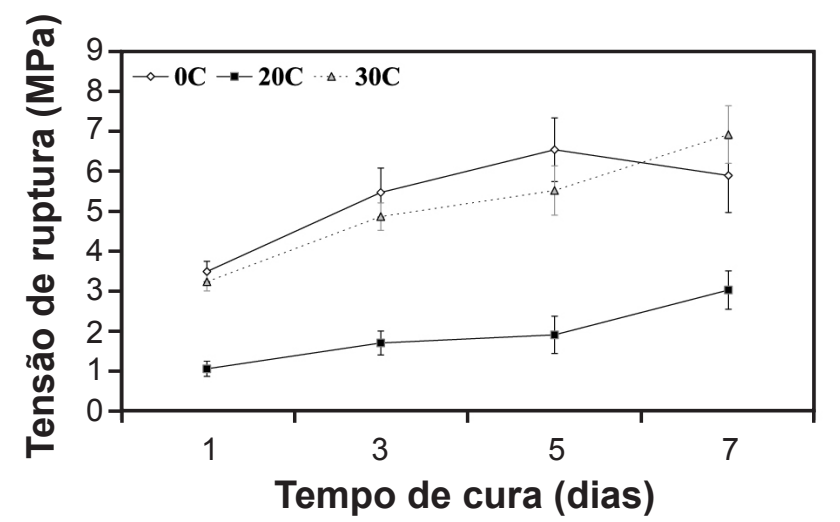

Figura 1: Resistência mecânica em função do tempo de cura para os concretos ligados por alumina hidratável (0C) ou cimentos de aluminato de cálcio distintos $(20 \mathrm{C}-20 \%-\mathrm{p} \mathrm{CaO}$ e $30 \mathrm{C}-30 \%-\mathrm{p}$ $\mathrm{CaO})$.

[Figure 1: Mechanical strength as a function of curing time for castables bonded with hydratable alumina (OC) or different calcium aluminate cements (20C - $20 \mathrm{wt} . \% \mathrm{CaO}$ and $30 \mathrm{C}-30 \mathrm{wt} . \% \mathrm{CaO}$ ).]

substituição de cimento de aluminato de cálcio (30C) por alumina hidratável (0C) resultou na diminuição do pico de decomposição de brucita [13] (Fig. 2). Este efeito é atribuído à redução de pH propiciada pela adição de alumina hidratável, resultando em menor quantidade de hidroxilas disponível para a formação de hidróxido de magnésio. Adicionalmente, outra alternativa para o controle da hidratação é o uso de um cimento de aluminato de cálcio com maior teor de alumina (20C). Para este material, percebe-se uma maior velocidade na saída de água, em decorrência da sua maior permeabilidade (maior teor de água na mistura) e, como conseqüência, o pico de decomposição de brucita não foi detectado. Tal resultado pode indicar que a pressurização do vapor de água tem um papel importante na formação de brucita. Seria esperado que o maior teor de água usado na composição $20 \mathrm{C}$ resultaria em uma maior formação de

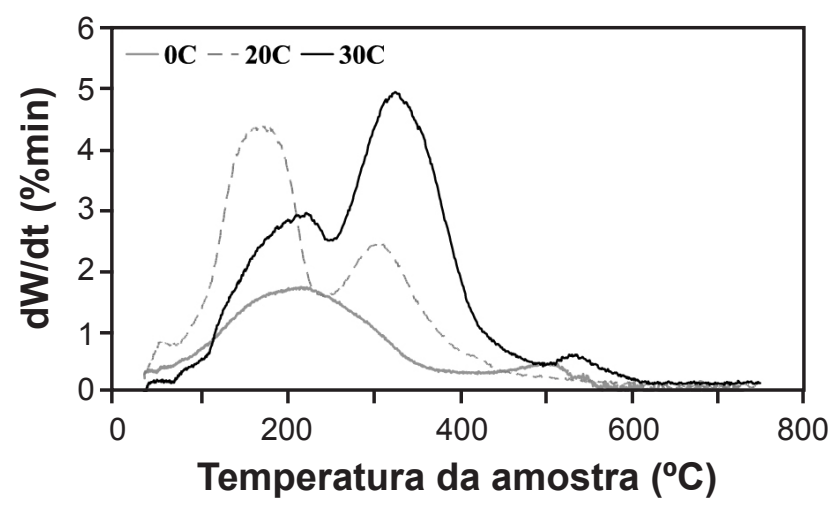

Figura 2: Taxa de secagem (dW/dt) em função da temperatura da amostra para os concretos ligados por alumina hidratável (0C) ou cimentos de aluminato de cálcio distintos (20C - 20\%-p CaO e 30C - 30\%-p CaO).

[Figure 2: Drying rate ( $d W / d t)$ as a function of sample temperature for castables bonded with hydratable alumina (OC) or different calcium aluminate cements (20C-20 wt $\% \mathrm{CaO}$ and $30 \mathrm{C}-30 \mathrm{wt} . \%$ $\mathrm{CaO})$.] 
brucita. No entanto, a maior permeabilidade do sistema (inferida pela saída de água a uma menor temperatura para a composição 20C) parece ter sido fundamental em inibir a formação desta fase.

A alumina hidratável (0C) apresentou problemas de resistência mecânica em temperaturas intermediárias, especialmente a $1000{ }^{\circ} \mathrm{C}$, como indica a Fig. 3, devido às transformações de fases da alumina [10]. Para o cimento de aluminato de cálcio tradicional (30C), observou-se uma queda de resistência mecânica até a temperatura de $600{ }^{\circ} \mathrm{C}$ em decorrência da decomposição dos seus hidratos, seguida por um acréscimo de seus valores de 600 a $1000{ }^{\circ} \mathrm{C}$, devido ao reaparecimento dos aluminatos de cálcio. Já o cimento de maior refratariedade (20C) apresentou baixos valores de resistência mecânica entre 110 e $800^{\circ} \mathrm{C}$, dada à sua menor quantidade de hidratos formados. No entanto, um aumento sutil na resistência foi observado de 800 para $1000{ }^{\circ} \mathrm{C}$ indicando o inicio de sua sinterização, devido à sua maior reatividade (maior teor de alumina reativa).

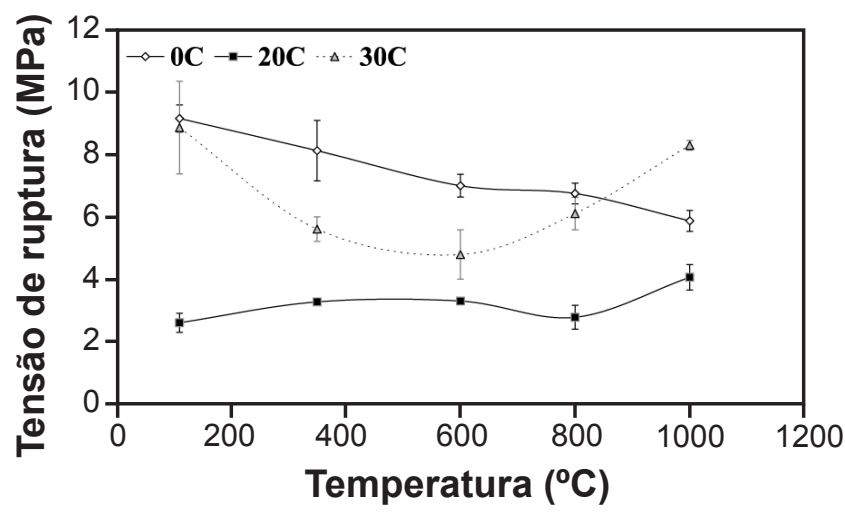

Figura 3: Resistência mecânica em temperaturas intermediárias para os concretos ligados por alumina hidratável (0C) ou cimentos de aluminato de cálcio distintos (20C - 20\%-p CaO e 30C - 30\%-p $\mathrm{CaO})$.

[Figure 3: Mechanical strength at intermediate temperatures for castables bonded with hydratable alumina (OC) or different calcium aluminate cements (20C-20 wt.\% CaO and 30C-30 wt.\% CaO).]

A queda de resistência mecânica observada na composição com alumina hidratável (0C) refletiu na avaliação de tal propriedade após queima por $5 \mathrm{~h}$ a 1150,1300 e $1500{ }^{\circ} \mathrm{C}$. A Fig. 4 indica o pior desempenho deste material, quando comparado aos concretos ligados por CAC. Apesar deste resultado, observa-se um ganho na resistência mecânica com o aumento de temperatura, devido à capacidade de sinterização da alumina hidratável. Com relação aos cimentos, destacam-se os resultados obtidos para o 20C, material de maior resistência mecânica após queima a $1500{ }^{\circ} \mathrm{C}$. Este aspecto pode ser associado à maior sinterabilidade de tal material, quando comparado ao $30 \mathrm{C}$, devido ao seu maior teor de alumina. Adicionalmente, a presença de $\mathrm{CA}_{6}$ nos concretos contendo CAC pode contribuir para a melhor resistência mecânica em relação ao concreto ligado por alumina hidratável, desde que sua formação ocorra de modo controlado [11].

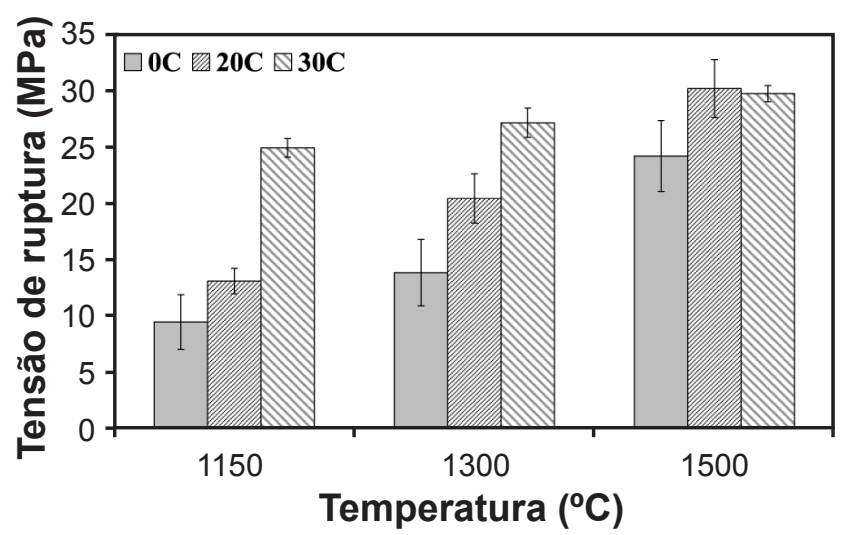

Figura 4: Resistência mecânica em função da temperatura de queima para os concretos ligados por alumina hidratável (0C) ou cimentos de aluminato de cálcio distintos (20C - 20\%-p CaO e $30 \mathrm{C}$ - 30\%-p CaO).

[Figure 4: Mechanical strength as a function of firing temperature for castables bonded with hydratable alumina (OC) or different calcium aluminate cements (20C-20 wt.\% CaO and 30C-30 wt.\% $\mathrm{CaO}$.]

A formação de hexaluminato de cálcio afetou de forma relevante a estabilidade volumétrica de concretos aluminamagnésia, devido ao seu caráter expansivo. Na Fig. 5 observa-se que a expansão linear aumenta com o aumento do teor de $\mathrm{CaO}$, indicando que o comportamento de expansão dos concretos alumina-magnésia depende não somente da formação de espinélio, mas também do tipo de ligante empregado.

A diminuição da expansão ocasionada pelo uso de alumina hidratável pode ser associada a três aspectos: 1) maior densificação propiciada pelo uso de @-alumina quando comparada à $\alpha$-alumina [14], 2) à retração associada a este tipo de ligante, devido à transformação da fase @ para $\alpha$ alumina e pela formação de um líquido metaestável entre a

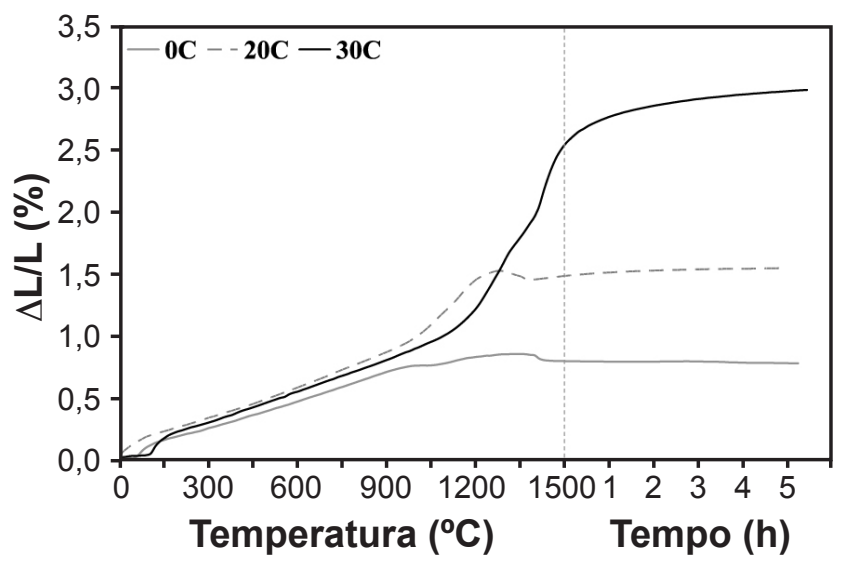

Figura 5: Variação dimensional em função da temperatura e do tempo para os concretos ligados por alumina hidratável (0C) ou cimentos de aluminato de cálcio distintos (20C - 20\%-p CaO e 30C - 30\%-p CaO).

[Figure 5: Dimensional change as a function of temperature and time for castables bonded with hydratable alumina (OC) or different calcium aluminate cements (20C - $20 \mathrm{wt} \% \mathrm{CaO}$ and $30 \mathrm{C}$ - 30 wt.\% CaO).] 
sílica e a alumina [15] e 3) a ausência de expansão decorrente da formação de $\mathrm{CA}_{6}$. Tais aspectos são evidenciados pelas derivadas das curvas de expansão, observadas na Fig. 6. Nesta figura, verifica-se uma taxa de variação linear negativa para a alumina hidratável $(0 \mathrm{C})$, indicando que a retração do sistema prevalece sobre a expansão propiciada pela espinelização in-situ. Para o cimento de aluminato de cálcio de menor refratariedade (30C), dois picos de expansão são evidenciados e podem ser atribuídos à formação de espinélio e $\mathrm{CA}_{6}$, como indicam estudos anteriores dos autores [11, 12, 16]. Para o CAC de menor teor de $\mathrm{CaO}$ (20C), observa-se um pico de espinélio de menor intensidade, indicando que a alumina reativa presente em tal material pode contribuir para a acomodação da espinelização in-situ. Adicionalmente, a maior sinterabilidade e o menor teor de $\mathrm{CaO}$ deste sistema não permitiu a detecção do pico de $\mathrm{CA}_{6}$. Os distintos comportamentos observados para cada um destes ligantes evidenciam possibilidades para o controle da expansão de concretos alumina-magnésia.

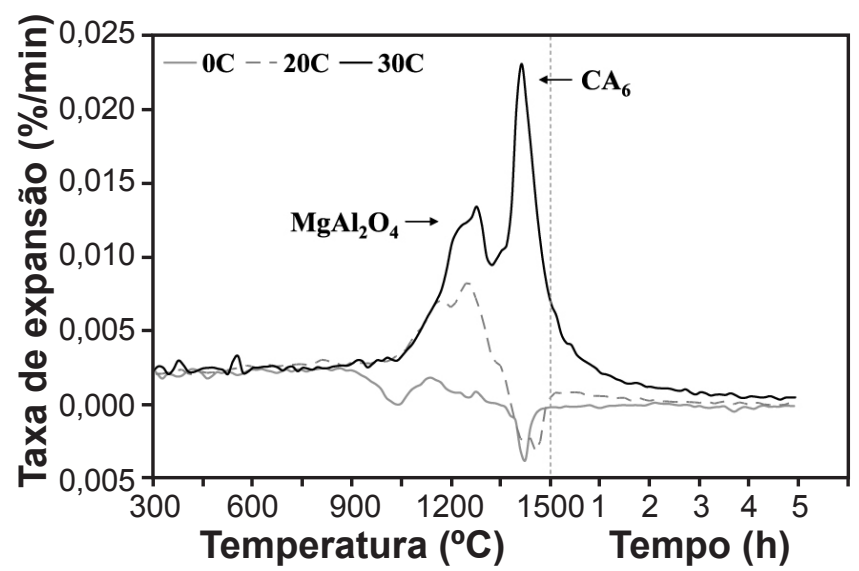

Figura 6: Taxa de expansão em função da temperatura e do tempo para os concretos ligados por alumina hidratável (0C) ou cimentos de aluminato de cálcio distintos (20C - 20\%-p CaO e 30C - 30\%-p $\mathrm{CaO})$.

[Figure 6: Expansion rate as a function of temperature and time for castables bonded with hydratable alumina (OC) or different calcium aluminate cements (20C-20 wt.\% CaO and $30 \mathrm{C}-30 \mathrm{wt} . \%$ $\mathrm{CaO})$.]

\section{CONCLUSÕES}

Os ligantes utilizados neste estudo alteraram $\mathrm{o}$ comportamento de expansão de concretos $\mathrm{Al}_{2} \mathrm{O}_{3}-\mathrm{MgO}$. Tais materiais apresentaram respostas distintas durante as etapas de processamento (cura, secagem, em temperaturas intermediárias e sinterização). Um elevado teor de $\mathrm{CaO}(30 \%$ p) foi benéfico para a resistência mecânica. No entanto, tal material resultou no maior pico de decomposição de brucita e na maior expansão observada durante a sinterização. $\mathrm{O}$ concreto contendo alumina hidratável apresentou um bom comportamento até o quinto dia de cura, seguido pela queda de resistência mecânica no sétimo dia, necessitando controle quando se quer inibir hidratação do $\mathrm{MgO}$. Em temperaturas intermediárias, observou-se uma queda na resistência mecânica, principalmente a $1000{ }^{\circ} \mathrm{C}$. Tal problema refletiu na etapa de sinterização do material, resultando no pior desempenho mecânico quando comparado aos cimentos. Por outro lado, sua retração propiciou melhor controle da expansão decorrente da formação in situ de espinélio. O concreto ligado pelo CAC contendo 20\%-p de $\mathrm{CaO}$ se destacou em temperaturas elevadas, em decorrência da sua alta sinterabilidade. Devido a este aspecto e pelo menor teor de $\mathrm{CA}_{6}$ formado, a expansão do sistema pôde ser controlada. Deste modo, o presente trabalho indicou alternativas para o controle da expansão e avaliou o impacto dos ligantes utilizados no processamento de concretos alumina-magnésia, propiciando maior flexibilidade e materiais de desempenho elevado para aplicação em panelas de siderurgia.

\section{AGRADECIMENTOS}

À FIRE (Federation for International Refractory Research and Education), à Magnesita S.A. (Brasil) e à FAPESP pelo suporte a este trabalho.

\section{REFERÊNCIAS}

[1] J. R. Garcia, I. R. Oliveira, V. C. Pandolfelli, Processo de hidratação e os mecanismos de atuação de aditivos aceleradores e retardadores de pega do cimento de aluminato de cálcio, Cerâmica 53, 325 (2007) 42.

[2] F. A. Cardoso, M. D. M. Innocentini, M. M. Akiyoshi, V. C. Pandolfelli, Effect of curing conditions on the properties of ultra-low cement refractory castables, Refractories Applications News 9, 2 (2004) 12.

[3] ALCOA, High alumina cements and chemical binders, apostila do seminário "Refractories Technology - an Introduction and Update", Institute of Refractories Engineering, África do Sul (1996).

[4] N. Zhou, M. Liu, S. Zhang, Unified International Conference on Refractories, The Am. Ceram. Soc., Orlando, EUA (2005) 40.

[5] M. Madono, Alumina raw materials for the refractory industry, CN-Refractories 6, 3 (1999) 54-63.

[6] R. P. Racher, R. Kockegey-Lorenz, G. Büchel, A. Buhr, D. Gierisch, Unified Int. Conf. Refractories, The Am. Ceram. Soc., Orlando, EUA (2005) 402.

[7] Y. Hongo, $\rho$-alumina bonded castable refractories, Taikabutsu Overseas 40, 4 (1988) 226.

[8] G. Ye, T. Troczynski, Effect of magnesia on strength of hydratable alumina-bonded castable refractories, J. Mater. Sci. 40, 15 (2005) 3921.

[9] R. Salomão, M. R. Ismael, V. C. Pandolfelli, IV Encontro de Refratraristas e Usuários de Refratários, Assoc. Bras. Cerâmica, Teresópolis, RJ (2006).

[10] A. Altun, Unified International Conference on Refractories, The Am. Ceram. Soc., Orlando, EUA (2005) 134.

[11] M. A. L. Braulio, D. H. Milanez, E. Y. Sako, L. R. M. Bittencourt, V. C. Pandolfelli, Expansion behavior of 
cement-bonded alumina-magnesia refractory castables, Am. Ceram. Soc. Bull. 86, 12 (2007) 9201.

[12] M. A. L. Braulio, L. R. M. Bittencourt, J. Poirier, V. C. Pandolfelli, Microsilica effects on cement bonded aluminamagnesia refractory castables, J. Technical Association Refractories, Japan 28, 3 (2008) 180.

[13] M. A. L. Braulio, D. H. Milanez, E. Y. Sako, L. R. M. Bittencourt, V. C. Pandolfelli, Unified International Technical Conference on Refractories, German Refractories Association, Dresden, Alemanha (2007) 540.
[14] Z. Zhang, N. Li, Effect of polymorphism of $\mathrm{Al}_{2} \mathrm{O}_{3}$ on the synthesis of magnesium aluminate spinel, Ceram. Int. 31 (2005) 583.

[15] S. H. Risbud, J. A. Pask, $\mathrm{SiO}_{2}-\mathrm{Al}_{2} \mathrm{O}_{3}$ metastable phase equilibrium diagram without mullite, J. Mater. Sci. 13, (1978) 2449.

[16] M. A. L. Braulio, J. F. R. Castro, C. Pagliosa, L. R. M. Bittencourt, V. C. Pandolfelli, From macro to nano magnesia: designing the in-situ spinel expansion, J. Am. Ceram. Soc. 91, 9 (2008) 3090.

(Rec. 11/05/2008, Ac. 18/12/2008). 